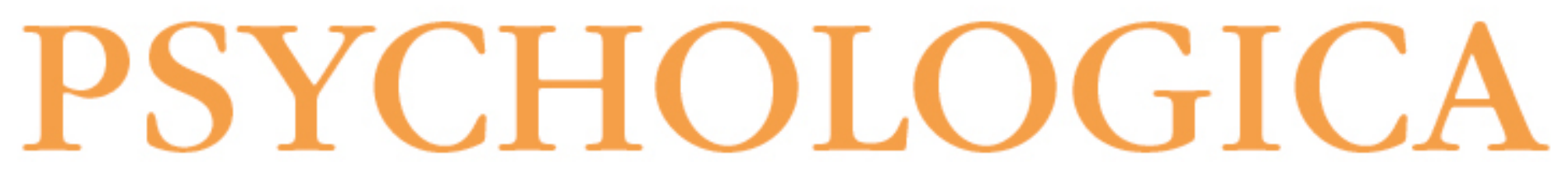

\title{
Problemas emocionais e comportamentais em jovens: relações com o temperamento, as estratégias de coping e de regulação emocional e a identificação de expressões faciais
}

Autor(es): $\quad$ Conceição, Andreia; Carvalho, Marina

Publicado por: Imprensa da Universidade de Coimbra

URL

persistente: URI:http://hdl.handle.net/10316.2/29957

DOI: DOI:http://dx.doi.org/10.14195/1647-8606_56_5

Accessed : $\quad$ 26-Apr-2023 13:21:00

A navegação consulta e descarregamento dos títulos inseridos nas Bibliotecas Digitais UC Digitalis, UC Pombalina e UC Impactum, pressupõem a aceitação plena e sem reservas dos Termos e Condições de Uso destas Bibliotecas Digitais, disponíveis em https://digitalis.uc.pt/pt-pt/termos.

Conforme exposto nos referidos Termos e Condições de Uso, o descarregamento de títulos de acesso restrito requer uma licença válida de autorização devendo o utilizador aceder ao(s) documento(s) a partir de um endereço de IP da instituição detentora da supramencionada licença.

Ao utilizador é apenas permitido o descarregamento para uso pessoal, pelo que o emprego do(s) título(s) descarregado(s) para outro fim, designadamente comercial, carece de autorização do respetivo autor ou editor da obra.

Na medida em que todas as obras da UC Digitalis se encontram protegidas pelo Código do Direito de Autor e Direitos Conexos e demais legislação aplicável, toda a cópia, parcial ou total, deste documento, nos casos em que é legalmente admitida, deverá conter ou fazer-se acompanhar por este aviso.

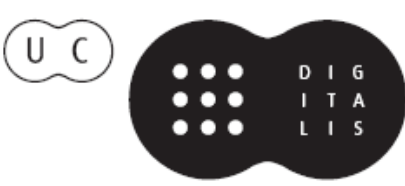


VOLUME 2013

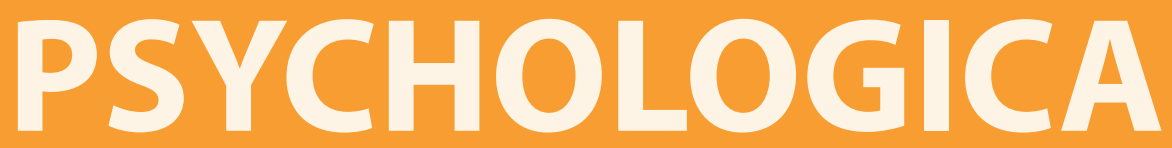

IMPRENSA DA UNIVERSIDADE DE COIMBRA

COIMBRA UNIVERSITY PRESS

FACULDADE DE PSICOLOGIA E DE CIÊNCIAS

DA EDUCAÇÃO DA UNIVERSIDADE DE COIMBRA 


\title{
Problemas emocionais e comportamentais em jovens: Relações com o temperamento, as estratégias de coping e de regulação emocional e a identificação de expressões faciais
}

\author{
Andreia Conceição e Marina Carvalho²
}

Emotional and behavioral problems in preadolescents:

Relationships with temperament, coping and emotional regulation strategies, and facial expressions identification

\begin{abstract}
With the main purpose of studying the relationships between temperament, coping and emotional regulation strategies with emotional and behavioral problems, a sample of 230 pre-adolescents was studied, 118 males and 112 females, mean age of 11 years old (SD $=0.81$ ), in the $5^{\text {th }}$ and $6^{\text {th }}$ school grades. Results have shown sex differences in affiliation (temperament dimension), in the frequency and efficacy of active and acting-out coping strategies, and in behavior problems and prosocial behavior. Effortful control, negative affectivity and negative emotional regulation were found to be common predictor factors of the different types of difficulties. The results were, in general, according to as expected and were discussed, based on the literature, theoretical models and taking into account their implications to assessment, prevention and intervention on emotional and behavioral problems in childhood and adolescence.
\end{abstract}

1 Universidade Lusófona de Humanidades e Tecnologias. E-mail: andreiafconceicao@gmail.com

2 Universidade Lusófona de Humanidades e Tecnologias/Instituto Superior Manuel Teixeira Gomes; Centro Hospitalar do Barlavento Algarvio; Centro para a Malária e Doenças Tropicais, LA 
Key-words: emotional and behavioral problems; temperament; coping strategies; emotional regulation; childhood and adolescence

\section{Resumo}

Com o objetivo geral de analisar as relações entre o temperamento, as estratégias de coping e de regulação emocional com os problemas emocionais e comportamentais, foi estudada uma amostra de 230 jovens, 118 do sexo masculino e 112 do sexo feminino, com uma média de idades igual a 11 anos $(D P=0.812)$, que frequentavam o $5^{\circ}$ e o $6^{\circ}$ anos de escolaridade. Os resultados obtidos demonstraram a existência de diferenças de sexo na dimensão do temperamento relativa à afiliação, na frequência e eficácia das estratégias ativas e de acting-out e nos problemas de comportamento e comportamento prosocial. $\mathrm{O}$ controlo com esforço, a afetividade negativa e a regulação emocional negativa mostraram ser fatores preditores comuns aos diferentes tipos de dificuldades. Os resultados obtidos estiveram, no geral, de acordo com o esperado e foram discutidos, com base na literatura e nos modelos explicativos neste âmbito e tendo em consideração as suas implicações para a avaliação, prevenção e intervenção nos problemas emocionais e comportamentais na infância e adolescência.

Palavras-chave: problemas emocionais e comportamentais; temperamento; estratégias de coping; regulação emocional; jovens

\section{INTRODUÇÃO}

Os problemas emocionais e comportamentais apresentam elevada prevalência na infância e adolescência (e.g., Merikangas, et al., 2010) e um duplo padrão em termos de sexo, com os rapazes a apresentarem uma maior tendência para o desenvolvimento de problemas de comportamento ou relacionados com o uso de substâncias, enquanto as raparigas apresentam uma tendência para o desenvolvimento de problemas emocionais (Borges, Manso, Tomé, \& Matos, 2008; Chaplin, Gillham, \& Selligman, 2009; Merikangas, et al., 2010; Roy, Groholt, Heyerdahl, \& Clench-Aas, 2006; Young, Sabbah, Young, Reiser, \& Richardson, 2010).

O desenvolvimento, a manutenção e a modificação, ao longo do tempo, dos problemas emocionais e comportamentais podem ser influenciados por diversos fatores, a nível individual, familiar e ambiental (Carvalho, 2007). Estudos efetuados neste âmbito têm demonstrado que crianças com um temperamento difícil, caracterizado, em particular, por baixos níveis de controlo com esforço, um processo 
de auto-regulação emocional que pode ser definido como a capacidade para inibir uma resposta dominante de modo a realizar uma resposta alternativa (Rothbart \& Bates, 2006; Rothbart \& Rueda, 2005), apresentam maior vulnerabilidade para o desenvolvimento de problemas emocionais e comportamentais (Eisenberg, et al., 2009; Letcher, Sanson, Smart, \& Toumbourou, 2012; Muris \& Ollendick, 2005; Nigg, 2006; Pitzer, Jennen-Steinmetz, Esser, Schmidt, \& Laucht, 2011). Por outro lado, as estratégias utilizadas para lidar com situações indutoras de stress, nomeadamente as estratégias ativas, focadas no problema, têm demonstrado ser um fator protetor do desenvolvimento de problemas emocionais e comportamentais enquanto as estratégias de coping de evitamento têm demonstrado relacionar-se diretamente com maior sintomatotologia emocional e comportamental (Compas, Connor-Smith, Saltzman, Thomsen, \& Wadsworth, 2001; Zalewski, Lengua, Wilson, Trancik, \& Bazinet, 2011a), da mesma forma que as estratégias de regulação emocional negativas (como a auto-culpabilização, a catastrofização, a ruminação e a baixa re-avaliação positiva) têm apresentado uma forte relação com os problemas de internalização, enquanto a menor utilização de estratégias de regulação emocional positivas, como a re-orientação positiva e a elevada utilização de catastrofização e culpabilização dos outros, se tem relacionado com os problemas de externalização (Garnefski, Kraaij, \& von Etten, 2005; Granic et al., 2012; Zalewski, Lengua, Wilson, Trancik, \& Bazinet, 2011b). Ainda, a regulação emocional está relacionada com a forma como a informação do meio envolvente é percebida, ou seja, o processamento da informação social parece influenciar a regulação emocional. Alguns destes processos, nomeadamente as dificuldades na identificação e reconhecimento de expressões faciais emocionais negativas, e a interpretação ambígua de situações negativas, para além de se relacionarem com o processo de regulação emocional, têm também demonstrado relações com os problemas emocionais e comportamentais (Ellis et al., 1997; Jarro et al., 2012; Muñoz, 2009; Schepman, Taylor, Collishaw, \& Fombonne, 2012).

Ainda que existam vários estudos que analisam a relação entre o temperamento, a regulação emocional e as estratégicas de coping com os problemas emocionais e comportamentais, não se conhece nenhum em particular que analise em simultâneo a relação entre todas estas variáveis com os diferentes tipos de problemas emocionais e comportamentais. Deste modo, uma vez que a prevalência dos problemas emocionais e comportamentais em jovens é elevada (Merikangas, et al., 2010) e que o desenvolvimento destes problemas está relacionado com fatores individuais, o presente estudo assume particular relevância, na medida em que pode contribuir para aprofundar a compreensão das relações entre estas variáveis individuais (temperamento, regulação emocional, estratégias de coping e identificação de expressões faciais) com problemas emocionais e comportamentais e, em 
consequência, facilitar o desenvolvimento de programas empiricamente baseados, com vista à sua prevenção, pela modificação dos fatores que estão associados ao seu desenvolvimento.

Assim, o presente estudo teve como objetivo geral a análise dos fatores preditores (de entre as dimensões do temperamento, da regulação emocional, das estratégias de coping e do reconhecimento das expressões faciais) dos problemas emocionais e comportamentais em jovens. Era esperado que os jovens que relatassem menos controlo com esforço, mais estratégias de regulação emocional negativas e mais estratégias de coping inadaptativas (que não visam a resolução do problema) apresentassem mais problemas emocionais e comportamentais.

\section{MÉTODo}

\section{Participantes}

Participaram neste estudo 230 jovens, 118 do sexo masculino e 112 do sexo feminino, com uma média de idades igual a 11 anos $(D P=0.81)$, que frequentavam o $5^{\circ}$ e $6^{\circ}$ anos de escolaridade. Não foram obtidas diferenças de sexo estatisticamente significativas para as variáveis em estudo, $p>.05$.

\section{Medidas}

Os problemas emocionais e comportamentais foram avaliados pela versão de autoavaliação do Strengths and Difficulties Questionnaire (SDQ) (Goodman, Meltzer, \& Bailey, 1998; versão portuguesa de Fleitlich, Loureiro, Fonseca, \& Gaspar, 2004), um questionário constituído por 25 itens, com um formato de resposta numa escala de tipo Likert de três pontos (0. Não é verdade; 2. É muito verdade) que se agrupam em cinco dimensões: Sintomas Emocionais, Problemas de Comportamento, Hiperatividade, Problemas de Relacionamento com os Pares e Comportamento prosocial. A medida permite, ainda, obter uma nota relativa ao total de dificuldades, obtida pela soma dos resultados obtidos em todas as dimensões, exceto o comportamento prosocial. A versão original apresenta valores de consistência interna satisfatórios, com valores a Cronbach entre .61 (Problemas de Relacionamento com os Pares) e .75 (Sintomas Emocionais) (Goodman, et al., 1998). Por sua vez, a 
versão portuguesa também demonstra qualidades psicométricas adequadas, com valores de consistência interna semelhantes, e superiores em algumas dimensões, aos da versão original (Marzocchi et al., 2004). Na amostra em estudo, foram obtidos valores de consistência interna, a Cronbach, que variaram entre .51 para os Problemas de Relacionamento com os Pares e .74 para o Total de Dificuldades.

O temperamento foi avaliado através do Early Adolescent Temperament Questionnaire (EATQ-R), (Ellis \& Rothbart, 2001, tradução de Carvalho, 2007), um questionário de autoavaliação das dimensões do temperamento de acordo com o modelo de Capaldi e Rothbart (1992), composto por 65 itens com um formato de resposta numa escala de tipo Likert de cinco pontos (1. Quase nunca se aplica; 5 Quase sempre se aplica). O EATQ-R possui doze dimensões que podem ser agrupadas em quatro fatores principais do temperamento: controlo com esforço (capacidade para realizar uma ação, quando há uma forte tendência para evitá-la), afiliação (desejo de proximidade com os outros, capacidade de focar a atenção), afetividade negativa (caracterizada pela frustração, humor depressivo e ações agressivas) e extroversão (baixos níveis de timidez e de medo). Na versão original, foram obtidos valores adequados de consistência interna, a de Cronbach entre 65 (Medo) e .82 (Timidez), tendo também sido obtidas evidências a propósito da sua validade convergente (Ellis \& Rothbart, 2001). O estudo das qualidades psicométricas da versão portuguesa da medida evidenciou valores de consistência interna adequados, a de Cronbach, entre .68 (Humor Depressivo) e .92 (Autorregulação), tendo a medida apresentado correlações no sentido esperado com uma medida de problemas emocionais e comportamentais (Conceição, et al., 2011). No presente estudo, esta medida apresentou valores de consistência interna, a Cronbach, que variaram entre . 45 (Extroversão) e .75 (Afiliação).

A regulação emocional foi avaliada através da versão reduzida do Cognitive Emotion Regulation Questionnaire (CERQ), (Garnefski, Kraaij, \& Spinhoven, 2001, versão portuguesa de Andrade et al., 2011), um questionário de autoavaliação das estratégias cognitivas de regulação emocional utilizadas em situações difíceis. O CERQ é composto por 19 itens com um formato de resposta numa escala de tipo Likert de cinco pontos (1. Nunca; 5. Quase sempre), que se agrupam em nove dimensões: auto-culpabilização, culpabilização dos outros, ruminação, catastrofização, perspetivação, re-orientação positiva, re-avaliação positiva, aceitação e planeamento (Garnefski \& Kraaij, 2006a). A versão reduzida original do CERQ apresenta, no geral, valores de consistência interna adequados, a de Cronbach entre .68 (Auto-culpabilização) e .81 (Re-avaliação Positiva e Catastrofização). A medida mostra ter validade concorrente, correlacionando-se positivamente com medidas de autoavaliação de depressão e ansiedade (Garnefski \& Kraaij, 2006b). Na versão portuguesa, o CERQ também apresentou valores de consistência interna adequados, 
a de Cronbach entre .68 (Catastrofização) e .81 (Atribuições e Foco na Mudança), apresentando ainda validade concorrente com uma medida de autoavaliação dos problemas emocionais e comportamentais (Andrade, et al., 2011). No presente estudo, foram consideradas duas dimensões do CERQ: regulação emocional negativa (auto-culpabilização, ruminação, catastrofização e culpabilização dos outros) e regulação emocional positiva (aceitação, re-avaliação positiva, re-orientação positiva, planeamento e perspetivação), que apresentaram valores de consistência, a Cronbach, de .62 e .73, respetivamente.

As estratégias de coping foram avaliadas através do Schoolagers' Coping Strategies Inventory (SCSI), (Ryan-Wenger, 1990; versão portuguesa de Lima, Lemos, \& Guerra, 2002), um questionário de autoavaliação da perceção dos jovens acerca da utilização de estratégias de coping quando confrontados com acontecimentos geradores de stress. A versão portuguesa do SCSI é constituída por 21 itens com um formato de resposta numa escala de tipo Likert de quatro pontos, que avalia a frequência da utilização de diferentes estratégias de coping (0. Nunca; 3. A maior parte das vezes) e a eficácia dessas estratégias (0. Nunca faço isto; 3. Ajuda muito). O SCSI permite obter três dimensões, com duas vertentes cada, frequência e eficácia, relativas a três tipos de estratégias de coping: distração cognitivo-comportamental (estratégias que visam a distração da situação de stress) acting-out (ou exteriorização de afetos negativos) e ativas (estratégias que estão centradas nos recursos internos dos jovens e que visam a resolução da situação). Na versão original, o SCSI foi considerado como uma medida unidimensional, tendo apresentado um valor de consistência interna, a de Cronbach, adequado, igual a .79, e uma validade concorrente baixa (Ryan-Wenger, 1990). A versão portuguesa apresentou valores de consistência interna satisfatórios, a de Cronbach iguais a .77. para ambas as escalas, e entre .53 (estratégias ativas) e .78 (distração cognitiva e comportamental e estratégias de acting-out) quando consideradas as diferentes dimensões (Lima, et al., 2002). Na amostra em estudo, foram verificados valores de consistência interna, $\alpha$ Cronbach, que variaram entre .60 para as estratégias ativas e .79 para as estratégias de acting-out.

O reconhecimento de expressões faciais emocionais foi avaliado através da apresentação, em papel, de oito estímulos representados por oito faces esquemáticas, desenvolvidas no âmbito do projeto “Trajetórias Desenvolvimentais dos Problemas Emocionais e Comportamentais em Jovens" (a decorrer) que mostram oito emoções diferentes (negativas e positivas: ira, surpresa, alegria, desapontamento, felicidade, vergonha, tristeza e medo), sendo pedido aos jovens que observem atentamente cada imagem e que identifiquem cada uma das emoções que são apresentadas. As respostas corretas em cada uma das expressões faciais são somadas de modo a calcular duas notas totais, relativas às expressões faciais negativas e positivas corretamente reconhecidas. 


\section{Procedimento}

Após o estabelecimento de um contacto inicial com algumas escolas, com o objetivo de perceber a sua disponibilidade para a realização do presente estudo, foi formalizado, por escrito, às escolas que manifestaram interesse, o pedido de autorização aos Presidentes dos respetivos Conselhos Diretivos para a recolha de dados no âmbito do presente estudo. Posteriormente, e através dos Diretores de Turma, foi solicitado o pedido de consentimento informado aos pais dos estudantes. Depois de cedida a autorização pelos encarregados de educação, o protocolo (constituído por uma folha de dados demográficos, o EATQ-R, o CERQ, o SCSI, o SDQ e pelas expressões faciais) foi administrado aos jovens, em contexto de grupo, durante as aulas de Formação Cívica, após a explicação dos objetivos do estudo, a garantia dos aspetos éticos de base ao mesmo e o seu próprio consentimento informado. Foram feitos, no total, 463 pedidos de consentimento informado aos progenitores, tendo sido devolvidos 274 (59\%). Destes, 42 não autorizaram a participação dos jovens no presente estudo e 2 protocolos foram excluídos da amostra por preenchimento incompleto do protocolo e por dificuldades de compreensão das questões colocadas.

\section{Análise dos Dados}

A análise dos dados foi feita através do SPSS, versão 18.0, para o Windows. As comparações de sexo foram analisadas através de testes t de Student para amostras independentes e as análises dos fatores preditores dos problemas emocionais e comportamentais foram executadas através de análises de regressão linear múltiplas, pelo método stepwise, nas quais foram incluídas como variáveis independentes as dimensões que na análise de correlações mostraram relacionar-se de forma significativa com os problemas emocionais e comportamentais

\section{RESULTADOS}

\section{Diferenças de sexo}

Com o objetivo de analisar as diferenças de sexo para as dimensões do temperamento, estratégias de coping e de regulação emocional, identificação de expressões 
faciais, e dos problemas emocionais e comportamentais, foram realizados teste $t$ de Student para amostras independentes (ver Tabela 1).

Tabela 1

Diferenças de Sexo para o Temperamento, Regulação Emocional, Estratégias de Coping, Identificação de Expressões Faciais e Problemas Emocionais e Comportamentais

\begin{tabular}{|c|c|c|c|c|c|}
\hline & \multicolumn{2}{|c|}{ Sexo Masculino } & \multicolumn{2}{|c|}{ Sexo Feminino } & \multirow[b]{2}{*}{$\mathrm{t}$} \\
\hline & $\mathrm{M}$ & DP & M & $\mathrm{DP}$ & \\
\hline Controlo com esforço & 53.05 & 6.79 & 53.71 & 8.33 & -.66 \\
\hline Extroversão & 49.37 & 6.28 & 50.46 & 7.08 & -1.24 \\
\hline Afiliação & 49.33 & 8.39 & 52.39 & 7.64 & $-2.89^{\star *}$ \\
\hline Afetividade Negativa & 53.14 & 8.43 & 54.70 & 9.75 & -1.30 \\
\hline Regulação emocional negativa & 24.22 & 5.19 & 23.34 & 4.38 & 1.40 \\
\hline Regulação emocional positiva & 34.79 & 6.13 & 34.99 & 6.21 & -.25 \\
\hline $\begin{array}{l}\text { Freq. Distração Cognitivo- } \\
\text {-Comportamental }\end{array}$ & 26.13 & 5.28 & 26.14 & 4.93 & -.02 \\
\hline Freq. Acting-out & 8.62 & 3.09 & 7.72 & 2.64 & $2.37^{\star}$ \\
\hline Freq. Estratégias ativas & 15.05 & 3.12 & 15.87 & 2.94 & $-2.05^{\star}$ \\
\hline $\begin{array}{l}\text { Efic. Distração Cognitivo-Com- } \\
\text { portamental }\end{array}$ & 26.11 & 4.65 & 26.73 & 4.19 & -1.04 \\
\hline Efic. Acting-out & 9.21 & 3.08 & 8.33 & 2.87 & $2.24^{\star}$ \\
\hline Efic. Estratégias ativas & 19.22 & 3.55 & 20.22 & 3.21 & $-2.22^{\star}$ \\
\hline Problemas emocionais & 3.92 & 2.10 & 4.45 & 2.07 & -1.91 \\
\hline Problemas de comportamento & 3.19 & 1.74 & 2.17 & 1.73 & $4.43^{\star * *}$ \\
\hline Hiperatividade & 4.76 & 1.95 & 4.21 & 2.09 & $2.09^{*}$ \\
\hline Relacionamento com os pares & 3.45 & 2.04 & 2.60 & 1.71 & $3.42^{\star * *}$ \\
\hline Comportamento Prosocial & 7.14 & 1.98 & 8.04 & 1.82 & $-3.56^{* * *}$ \\
\hline Total de dificuldades & 14.11 & 4.99 & 12.71 & 4.97 & $2.13^{\star}$ \\
\hline Expressões faciais positivas & .81 & .67 & .94 & .74 & -1.34 \\
\hline Expressões faciais negativas & 2.31 & 1.14 & 2.62 & 1.10 & $-2.05^{\star}$ \\
\hline
\end{tabular}

${ }^{*} \mathrm{p} \leq .05 ;{ }^{* *} \mathrm{p} \leq .01 ;{ }^{* *} \mathrm{p} \leq .001$.

No que respeita às dimensões do temperamento, apenas foi obtida uma diferença de sexo, estatisticamente significativa, na dimensão da afiliação, $t(228)=-2.89$, $p=.004$, com o sexo feminino a relatar níveis médios mais elevados de afiliação, comparativamente com o sexo masculino.

Para as dimensões das estratégias de coping, foram obtidas diferenças de sexo estatisticamente significativas na frequência de estratégias de acting-out, $t(228)=2.37, p=.02$, e de estratégias ativas, $t(228)=-2.05, p=.04$, e na eficácia de estratégias de acting-out, $t(228)=2.24, p=.03$, e de estratégias ativas, $t(228)$ $=-2.22, p=.03$. Os rapazes relataram utilizar com mais frequência e perceber 
como mais eficazes estratégias de coping de acting-out, enquanto as raparigas relataram utilizar com mais frequência e perceber como mais eficazes as estratégias de coping ativas.

No que se refere aos problemas emocionais e comportamentais, as diferenças de sexo estatisticamente significativas foram obtidas nas dimensões relativas aos problemas de comportamento, $t(228)=4.43, p=.0001$, hiperatividade, $t(228)=$ $2.09, p=.04$, problemas de relacionamento com os pares, $t(228)=3.42, p=.001$, comportamento prosocial, $t(228)=-3.56, p=.0001 \mathrm{e}$, ainda, no total de dificuldades, $t(228)=2.13, p=.03$. Os rapazes relataram mais dificuldades no geral e, especificamente, mais problemas de comportamento, mais hiperatividade e mais problemas de relacionamento com os pares, enquanto as raparigas relataram mais comportamentos prosociais.

Por fim, foram também verificadas diferenças de sexo estatisticamente significativas na identificação das expressões faciais negativas, $t(228)=-2.05, p=.04$, com as raparigas a identificarem mais expressões faciais negativas comparativamente com os rapazes.

Fatores preditores dos problemas emocionais e comportamentais

Com o objetivo de analisar, de entre as dimensões das variáveis individuais avaliadas que se correlacionaram com as diferentes dimensões dos problemas emocionais e comportamentais, quais as que os explicavam, foram realizadas análises de regressão linear múltiplas, pelo método stepwise, assumindo como variáveis independentes preditoras as dimensões do temperamento, da regulação emocional, das estratégias de coping e da identificação de expressões faciais, e como variáveis dependentes as dimensões dos problemas emocionais e comportamentais. As análises foram realizadas em função do sexo, tendo em consideração a existência de diferenças estatisticamente significativas para quase todas as dimensões em análise.

A Tabela 2 apresenta os modelos preditores obtidos para o sexo masculino (ver Tabela 2). 
Tabela 2

Fatores Preditores dos Problemas Emocionais e Comportamentais para o Sexo Masculino

\begin{tabular}{|c|c|c|c|c|c|}
\hline \multirow[b]{2}{*}{ Variáveis Independentes } & \multicolumn{5}{|c|}{ Problemas Emocionais } \\
\hline & $R^{2}$ & $R^{2}$ aj. & $\beta$ & $t$ & $F(d f)$ \\
\hline Frequência estratégias ativas & .17 & .16 & .36 & $3.96^{\star * *}$ & $23.62(1)$ \\
\hline Expressões faciais negativas & .20 & .19 & -.17 & $-2.14^{*}$ & $14.61(2)$ \\
\hline Regulação emocional positiva & .23 & .21 & -.26 & $-2.84^{\star \star}$ & $11.32(3)$ \\
\hline \multirow{2}{*}{ Regulação emocional negativa } & .27 & .25 & .25 & $2.61^{\star *}$ & $10.64(4)$ \\
\hline & \multicolumn{5}{|c|}{ Problemas de Comportamento } \\
\hline Frequência acting-out & .19 & .19 & .32 & $3.88^{* * *}$ & $28.72(1)$ \\
\hline Controlo com esforço & .29 & .28 & -.27 & $-3.48^{* * *}$ & $23.79(2)$ \\
\hline Afetividade negativa & .35 & .33 & .26 & $3.29^{* * *}$ & $20.32(3)$ \\
\hline \multirow[t]{2}{*}{ Eficácia distração cognitvo-comportamental } & .38 & .36 & -.17 & $-2.27^{\star}$ & $17.07(4)$ \\
\hline & \multicolumn{5}{|c|}{ Hiperatividade } \\
\hline Controlo com esforço & .11 & .10 & -.30 & $-3.45^{\star * *}$ & $13.96(1)$ \\
\hline \multirow[t]{2}{*}{ Afetividade negativa } & .15 & .14 & .22 & $2.50^{\star}$ & $10.42(2)$ \\
\hline & \multicolumn{5}{|c|}{ Relacionamento com os pares } \\
\hline Controlo com esforço & .11 & .11 & -.27 & $-3.12^{\star *}$ & $14.71(1)$ \\
\hline Regulação emocional negativa & .15 & .13 & .30 & $3.14^{\star *}$ & $9.82(2)$ \\
\hline \multirow[t]{2}{*}{ Regulação emocional positiva } & .19 & .17 & -.25 & $-2.62^{\star *}$ & $9.17(3)$ \\
\hline & \multicolumn{5}{|c|}{ Comportamento prosocial } \\
\hline Afiliação & .18 & .17 & .32 & $3.88^{* * *}$ & $25.48(1)$ \\
\hline Frequência estratégias ativas & .27 & .26 & .37 & $4.48^{\star * *}$ & $21.04(2)$ \\
\hline Frequência acting-out & .33 & .31 & -.25 & $-3.22^{\star *}$ & $18.64(3)$ \\
\hline
\end{tabular}

${ }^{*} \mathrm{p} \leq .05 ;{ }^{* *} \mathrm{p} \leq .01 ;{ }^{* *} \mathrm{p} \leq .001$.

Para os problemas emocionais, no caso dos rapazes, o modelo preditor obtido ficou composto por quatro variáveis independentes preditoras, frequência das estratégias ativas $(\beta=.36 ; t=3.96 ; p=.0001)$, expressões faciais negativas $(\beta=$ $-.17 ; t=-2.14 ; p=.04)$, regulação emocional positiva $(\beta=-.26 ; t=-2.84 ; p=$ $.01)$ e regulação emocional negativa $(\beta=.25 ; t=2.61 ; p=.01)$, que, no seu todo, explicaram $25 \%$ da variância total. Já o modelo preditor obtido para os problemas de comportamento, nos rapazes, ficou também composto por quatro variáveis independentes preditoras, frequência de acting-out $(\beta=.32 ; t=3.88 ; p=.0001)$, controlo com esforço $(\beta=-.27 ; t=-3.48 ; p=.001)$, afetividade negativa $(\beta=.26$; $t=3.29 ; p=.001)$ e eficácia da distração cognitivo-comportamental $(\beta=-.17 ; t$ $=-2.27 ; p=.03$ ), que no total, explicaram $36 \%$ da variância total. Na dimensão de hiperatividade, no sexo masculino, foram duas as variáveis independentes preditoras, controlo com esforço $(\beta=-.30 ; t=-3.45 ; p=.001)$ e afetividade negativa ( $\beta$ $=.22 ; t=2.50 ; p=.01)$ que explicaram, no seu conjunto, $14 \%$ da variância total. Também no que respeita aos problemas de relacionamento com os pares, o modelo 
preditor, para os rapazes, ficou constituído por três variáveis independentes que explicaram $17 \%$ da variância: controlo com esforço $(\beta=.27 ; t=-3.12 ; p=.002)$, regulação emocional negativa $(\beta=.30 ; t=3.14 ; p=.002)$ e regulação emocional positiva $(\beta=-.25 ; t=-2.62 ; p=.01)$. Por fim, foram três as variáveis independentes preditoras do comportamento prosocial, no sexo masculino, afiliação $(\beta=.32 ; t$ $=3.88 ; p=.0001)$, frequência de estratégias ativas $(\beta=.37 ; t=4.48 ; p=.0001) \mathrm{e}$ frequência de acting-out ( $\beta=-.25 ; t=-3.22 ; p=.002)$ que, no global, explicaram $31 \%$ da variância total. A Tabela 3 apresenta agora os fatores preditores obtidos para as diferentes dimensões dos problemas emocionais e comportamentais para o sexo feminino (ver Tabela 3 ).

Tabela 3

Fatores Preditores dos Problemas Emocionais e Comportamentais para o Sexo Feminino

\begin{tabular}{|c|c|c|c|c|c|}
\hline \multirow[b]{2}{*}{ Variáveis Independentes } & \multicolumn{5}{|c|}{ Problemas Emocionais } \\
\hline & $R^{2}$ & $R^{2} a j$. & $\beta$ & $t$ & $F(d f)$ \\
\hline Frequência estratégias ativas & .14 & .13 & .33 & $3.93^{* * *}$ & $17.17(1)$ \\
\hline Eficácia acting-out & .23 & .21 & .22 & $2.43^{\star}$ & $15.79(2)$ \\
\hline \multirow[t]{2}{*}{ Afetividade negativa } & .26 & .24 & .21 & $2.35^{\star}$ & $12.80(3)$ \\
\hline & \multicolumn{5}{|c|}{ Problemas de Comportamento } \\
\hline Frequência acting-out & .33 & .33 & .47 & $5.93^{* * *}$ & $55.25(1)$ \\
\hline Controlo com esforço & .39 & .38 & -.25 & $-3.12^{\star *}$ & $35.02(2)$ \\
\hline Expressões faciais positivas & .43 & .41 & .19 & $2.59^{\star}$ & $26.87(3)$ \\
\hline \multirow[t]{2}{*}{ Expressões faciais negativas } & .45 & .43 & -.16 & $-2.23^{*}$ & $22.13(4)$ \\
\hline & \multicolumn{5}{|c|}{ Hiperatividade } \\
\hline Controlo com esforço & .25 & .24 & -.36 & $-4.16^{\star * *}$ & $36.21(1)$ \\
\hline Frequência acting-out & .32 & .31 & .32 & $3.66^{* * *}$ & $26.14(2)$ \\
\hline \multirow[t]{2}{*}{ Expressões faciais positivas } & .35 & .33 & .16 & $2.09^{*}$ & $29.40(3)$ \\
\hline & \multicolumn{5}{|c|}{ Relacionamento com os pares } \\
\hline \multirow[t]{2}{*}{ Afetividade negativa } & .04 & .03 & .20 & $2.18^{*}$ & $4.74(1)$ \\
\hline & \multicolumn{5}{|c|}{ Comportamento prosocial } \\
\hline Eficácia estratégias ativas & .17 & .16 & .26 & $3.10^{\star *}$ & $21.79(1)$ \\
\hline Afiliação & .26 & .25 & .23 & $2.84^{\star *}$ & 19.21(2) \\
\hline Controlo com esforço & .32 & .30 & .27 & $3.37^{\star * *}$ & $17.15(3)$ \\
\hline Eficácia distração cognitivo-comportamental & .36 & .34 & .21 & $2.46^{*}$ & $14.97(4)$ \\
\hline
\end{tabular}

${ }^{*} \mathrm{p} \leq .05 ;{ }^{* *} \mathrm{p} \leq .01 ;{ }^{* *} \mathrm{p} \leq .001$.

Para os problemas emocionais, no sexo feminino, o modelo preditor obtido foi composto por três variáveis independentes preditoras, frequência das estratégias ativas $(\beta=.33 ; t=3.93 ; p=.0001)$, eficácia acting-out $(\beta=.22 ; t=2.43 ; p=$ $.02)$, e afetividade negativa $(\beta=.21 ; t=2.35 ; p=.02)$, que, no seu todo, explicaram $24 \%$ da variância total. O modelo preditor obtido para os problemas de 
comportamento, nas raparigas, ficou composto por quatro variáveis independentes preditoras, frequência de acting-out $(\beta=.47 ; t=5.93 ; p=.0001)$, controlo com esforço $(\beta=-.25 ; t=-3.12 ; p=.002)$, expressões faciais positivas $(\beta=.19 ; t=$ $2.59 ; p=.01)$ e expressões faciais negativas $(\beta=-.16 ; t=-2.23 ; p=.03)$, que no total, explicaram $43 \%$ da variância total. Na dimensão de hiperatividade, no sexo feminino, o modelo preditor obtido ficou constituído por três variáveis independentes preditoras, controlo com esforço $(\beta=-.36 ; t=-4.16 ; p=.0001)$, frequência de acting-out $(\beta=.32 ; t=3.66 ; p=.0001)$ e expressões faciais positivas $(\beta=.16 ; t$ $=2.09 ; p=.04$ ) que explicaram, no seu conjunto, $33 \%$ da variância total. No que respeita aos problemas de relacionamento com os pares, foi obtida uma variável independente, afetividade negativa $(\beta=.20 ; t=2.18 ; p=.03)$ que explicou $3 \% \mathrm{da}$ variância total, no caso das raparigas.

Por fim, no que respeita ao comportamento prosocial, o modelo preditor, para o sexo feminino, ficou constituído por quatro variáveis independentes que explicaram 34\% da variância:, eficácia das estratégias ativas $(\beta=.26 ; t=3.10 ; p=.003)$, afiliação $(\beta=.23 ; t=2.84 ; p=.01)$, controlo com esforço $(\beta=.27 ; t=3.37 ; p=$ $.001)$ e eficácia da distração cognitivo-comportamental $(\beta=.21 ; t=2.46 ; p=.02)$

\section{DISCUSSÃO}

O presente estudo pretendeu analisar a relação entre o temperamento, as estratégias de coping e de regulação emocional e o reconhecimento de expressões faciais com os problemas emocionais e comportamentais. Era esperado que os jovens que relatassem menos controlo com esforço, que utilizassem mais estratégias de regulação emocional negativas e mais estratégias de coping inadaptativas apresentassem mais problemas emocionais e comportamentais.

Dada a existência, na literatura, de diferenças de género em algumas das variáveis estudadas, as comparações entre sexos foram analisadas, tendo os resultados obtidos mostrado que as raparigas relataram valores mais elevados de afiliação, o que está de acordo com os resultados obtidos por Ellis e Rothbart (2001) e Muris e Meesters (2009). Também os resultados obtidos acerca das diferenças de sexo nas estratégias de coping estiveram de acordo com os obtidos nos estudos realizados por Raimundo e Pinto (2006) e por Lima e colaboradores (2002) e, no geral, as diferenças de sexo obtidas para os problemas emocionais e comportamentais estiveram também de acordo com a literatura (e.g., Roy, et al., 2006). Por fim, as diferenças de género obtidas no reconhecimento das expressões faciais foram de encontro aos resultados obtidos nos estudos realizados por McClure (2000) e Tottenham, Hare e Casey (2011). 
No entanto, e ao contrário do esperado, os resultados obtidos no presente estudo não evidenciaram a existência de diferenças de sexo estatisticamente significativas na regulação emocional. No estudo realizado por Garnefski e Kraaij (2006a), as adolescentes relataram valores superiores em todas as dimensões da regulação emocional cognitiva, sendo possível que os resultados agora obtidos possam ser explicados pelo modo como foram analisados os resultados obtidos pela medida, agrupados em apenas duas dimensões principais que, por esse facto, podem não demonstrar a existência de diferenças específicas em estratégias também específicas. Ainda especificamente no que respeita aos problemas emocionais, apesar de ser esperado que as raparigas apresentassem valores médios mais elevados (e.g., Borges, et al., 2008; Chaplin, et al., 2009; Merikangas, et al., 2010; Roy, et al., 2006; Young, et al., 2010), as diferenças obtidas não foram estatisticamente significativas, o que pode dever-se à natureza da amostra não clínica, mas também ao facto de, no presente estudo, a idade dos participantes ser inferior comparativamente com amostras utilizadas nos outros estudos (Merikangas, et al., 2010; Young, et al., 2010).

Os resultados obtidos pela análise dos fatores preditores das dimensões dos problemas emocionais e comportamentais estiveram também de acordo com a literatura, bem como com as hipóteses formuladas, e mostraram, que independentemente do sexo, os jovens com mais problemas emocionais relataram utilizar mais estratégias ativas, especificamente, os rapazes relataram também mais regulação emocional negativa, menos regulação emocional positiva, o que está de acordo com os outros estudos (eg., Garnefski, et al., 2001; 2005 Garnefski \& Kraaij, 2006a, Garnefski, Legerstee, Kraaij, van den Kommer, \& Teerds, 2002) e menor reconhecimento de expressões faciais negativas comparativamente com as raparigas com mais problemas emocionais que, de acordo com outros estudos (e.g., Calkins \& Fox, 2002; Lonigan \& Phillips, 2001) relataram mais afetividade negativa e maior eficácia das estratégias de acting-out também de acordo com investigações anteriores (Compas, et al., 2001; Holahan, Moos, \& Schaefer, 1996). Para os problemas de comportamento, em ambos os sexos, um menor controlo com esforço e uma maior utilização de estratégias de acting-out mostraram ser preditores dos problemas de comportamento, estando de acordo com outros estudos (Compas, et al., 2001; Holahan, Moos, \& Schaefer, 1996; Muris \& Ollendick, 2005; Nigg, 2006), especificamente, os jovens do sexo masculino relataram ainda mais afectividade negativa, de acordo com outras investigações (Calkins \& Fox, 2002; Lonigan \& Phillips, 2001) e menor eficácia das estratégias de distração cognitivo-comportamental quando comparados com o sexo feminino, para o qual o menor reconhecimento de expressões faciais negativas e maior reconhecimento de expressões positivas também se mostraram preditores dos problemas de comportamento. Em ambos os sexos, um menor controlo com esforço foi preditor de hiperatividade, tal como nos estudos de Muris e Ollendick 
(2005) e Nigg (2006). Especificamente os rapazes com mais hiperatividade também relataram mais afetividade negativa, tal como na literatura (Calkins \& Fox, 2002; Lonigan \& Phillips, 2001) e as raparigas com maior utilização de estratégias de acting-out, como nos estudos anteriores (Compas, et al., 2001; Holahan, Moos, \& Schaefer, 1996) e maior reconhecimento de expressões faciais positivas também apresentaram mais hiperatividade. Para o sexo masculino, menor controlo com esforço e regulação emocional positiva e maior regulação emocional negativa mostraram ser preditores dos problemas de relacionamento com os pares, indo de encontro aos estudos anteriores (Garnefski, et al., 2001; 2005; Garnefski \& Kraaij, 2006a; Garnefski, Legerstee, Kraaij, van den Kommer, \& Teerds, 2002; Muris \& Ollendick, 2005; Nigg, 2006;) comparativamente com o sexo feminino para o qual apenas a afetividade negativa mostrou prever estres problemas, sendo esta relação também confirmada na literatura (Calkins \& Fox, 2002; Lonigan \& Phillips, 2001).

Para ambos os sexos, uma maior afiliação foi preditora do comportamento prosocial, tal como na literatura (e.g., Eschenbeck, Kohlmann, \& Lohaus, 2007; Kochanska, Murray, \& Harlan, 2000; Muris \& Meesters, 2009; Rothbart \& Bates, 2006), especificamente os rapazes com maior utilização de estratégias ativas, menor utilização de estratégias de acting-out e as raparigas com maior controlo com esforço e maior eficácia das estratégias ativas e de distração cognitivo-comportamental também apresentaram mais comportamento prosocial, estando estes resultados de acordo com estudos anteriores (Compas, et al., 2001; Holahan et al., 1996), Muris \& Ollendick, 2005; Nigg, 2006.

Assim, e de um modo geral, os resultados do presente estudo mostraram que as raparigas relataram utilizar mais estratégias de coping ativas, mais afiliação e comportamento prosocial comparativamente com os rapazes que relataram mais estratégias de acting-out, mais problemas de comportamento, hiperatividade e mais problemas de relacionamento com os pares. No que respeita aos fatores preditores dos problemas emocionais e comportamentais, as hipóteses formuladas, no geral, foram confirmadas, no sentido em que os jovens que relataram menos controlo com esforço, mais regulação emocional negativa e maior utilização de estratégias de coping de acting-out relataram mais problemas emocionais e comportamentais. Apesar dos resultados obtidos, o presente estudo apresenta algumas limitações, relativas ao tamanho e natureza da amostra, não representativa e de conveniência, ao caráter transversal do estudo, que não permitiu estudar a evolução das variáveis ao longo do tempo. Neste sentido, sugerimos que estudos futuros analisem estas variáveis em amostras clínicas, em comparação com grupos subclínicos e não clínicos. Em estudos futuros, será, também, importante analisar as interações entre estas variáveis e, em particular o papel mediador das estratégias de coping e de regulação emocional na relação entre o temperamento e os problemas emocionais e 
comportamentais, bem como considerar o papel de outros fatores, como os estilos parentais, a vinculação, a existência de um histórico familiar de psicopatologia, que podem ser relevantes para explicar os principais fatores associados ao surgimento e manutenção dos problemas emocionais e comportamentais na infância e adolescência. Ainda, a avaliação da identificação e do reconhecimento emocional, bem como de outros processos cognitivos associados ao processamento de informação, através de provas não sujeitas a respostas socialmente desejáveis, pode contribuir para aprofundar o conhecimento acerca do papel dos enviesamentos no processamento de informação nos problemas emocionais e comportamentais.

Apesar das limitações que comporta, esperamos que os resultados obtidos no presente estudo possam contribuir para o conhecimento nesta área e, especificamente, para o desenvolvimento de programas de prevenção e/ou intervenção neste âmbito, que visem a promoção das estratégias de coping ativas e de regulação emocional positivas bem como de processos cognitivos (como a atenção e o processamento de informação) relacionados com o reconhecimento emocional que, em conjunto com as características do temperamento, podem contribuir para a diminuição deste tipo de dificuldades tão prevalentes na infância e adolescência.

\section{REFERÊNCIAS}

Andrade, C., Carvalho, M., Justino, M., Pacheco, S., Pacheco, S., \& Mendes, S. (2011, Julho). Questionário de Regulação Emocional Cognitiva para Jovens: Resultados preliminares das suas qualidades psicométricas na população portuguesa. Comunicação com o formato de Poster apresentada ao VIII Congresso Iberoamericano de Avaliação Psicológica / XV Conferência Internacional Avaliação Psicológica: Formas e Contextos da Faculdade de Psicologia da Universidade de Lisboa, Lisboa.

Borges, A., Manso, D., Tomé, G., \& Matos, M. (2008). Ansiedade e coping em crianças e adolescentes: Diferenças relacionadas com a idade e género. Análise Psicológica, 4(XXVI), 551-561.

Calkins, S. D., \& Fox, N. A. (2002). Self-regulatory process in early personality development: A multilevel approach to the study of childhood social withdrawal and aggression. Development and Psychopathology, 14, 477-498.

Capaldi, D. M., \& Rothbart, M. K. (1992). Development and Validation of an Early Adolescent Temperament Measure. Journal of Early Adolescence, 12(2), 153-173.

Carvalho, M. (2007). Vinculação, Temperamento e Processamento da Informação: Implicações nas Perturbações Emocionais e Comportamentais no início da Adolescência. (Tese de doutoramento). Disponível no Repositorium da Universidade do Minho em http:/hdl.handle.net/1822/8410.

Chaplin, T. M., Gillham, J. E., \& Selligman, M. E. P. (2009). Gender, Anxiety, and Depressive Symptoms: A longitudinal Study of Early Adolescents. Journal of Early Adolescence, 29(2), 307-327.

Compas, B. E., Connor-Smith, J. K., Saltzman, H., Thomsen, A. H., \& Wadsworth, M. E. (2001). Coping with stress during childhood and adolescence: Problems, progress, and potential in theory and research. Psychological Bulletin, 127, 87-127. 
Conceição, A., Carvalho, M., Justino, M., Pacheco, S., Pacheco, S., \& Mendes, S. (2011, Julho). Resultados Preliminares da Validação de uma Medida de Auto-Avaliação do Temperamento: O Questionário de Temperamento para Jovens - Revisto. Comunicação em formato de Poster apresentada ao VIII Congresso Iberoamericano de Avaliação Psicológica / XV Conferência Internacional Avaliação Psicológica: Formas e Contextos da Faculdade de Psicologia da Universidade de Lisboa, Lisboa.

Eisenberg, N., Valiente, C., Spinrad, T. L., Liew, J., Zhou, Q., Losoya, S. H.,..., Cumberland A., (2009). Longitudinal relations of children's effortful control, impulsivity, and negative emotionality to their externalizing, internalizing, and co-occurring behavior problems. Developmental Psychology, 45, 988-1008.

Ellis, C. R., Lindstrom, K. L., Villani, T. M., Singh, N. N., Best, A. M., Winton, A. S. W.,... Leung J.P.. (1997). Recognition of Facial Expressions of Emotion by Children with Emotional and Behavioral Disorders. Journal of Child and Family Studies, 6, 453-470.

Ellis, L. K., \& Rothbart, M. K. (2001). Revision of the Early Adolescent Temperament Questionnaire. Poster presented at the 2001 Biennial Meeting of the Society for Research in Child Development, Minneapolis, Minnesota.

Eschenbeck, H., Kohlmann, C. W., \& Lohaus, A. (2007). Gender Differences in Coping Strategies in Children and Adolescents. Journal of Individual Differences, 28(I), 18-26.

Fleitlich, B., Loureiro, M. J., Fonseca, A., \& Gaspar, F. (2004). Questionário do SDQ, versão traduzida e adaptada para a população portuguesa. Consultado em http://www.sdqinfo.com/d23.htlm).

Garnefski, N., \& Kraaij, V. (2006a). Relationships between cognitive emotion regulation strategies and depressive symptoms: A comparative study of five specific samples. Personality and Individual Differences, 40, 1659-1669.

Garnefski, N., \& Kraaij, V. (2006b). Cognitive emotion regulation questionnaire - development of a short 18-item version (CERQ-short). Personality and Individual Differences, 41, 1045-1053.

Garnefski, N., Kraaij, V., \& von Etten, M. (2005). Specificity of relations between adolescents' cognitive emotion regulation strategies and Internalizing and Externalizing psychopathology. Journal of Adolescence, 28, 619-631.

Garnefski, N., Kraaij, V., \& Spinhoven, P. (2001). Negative live events, cognitive emotion regulation and emotional problems. Personality and Individual Differences, 30, 1311-1327.

Garnefski, N., Legerstee, J., Kraaij, V., van den Kommer, T., \& Teerds, J. (2002). Cognitive coping strategies and symptoms of depression and anxiety: a comparison between adolescents and adults. Journal of Adolescence, 25, 603-611.

Goodman, R., Meltzer, H., \& Bailey, V. (1998). The Strengths and difficulties questionnaire: A pilot study on the validity of the self-report version. European Child \& Adolescent Psychiatry, 7, 125-130.

Granic, I., Meusel, L. A., Lamm, C., Woltering, S., \& Lewis, M. D. (2012). Emotion regulation in children with behavior problems: linking behavioral and brain processes. Developmental Psychopathology, 24, 1019-1029.

Holahan, C. J., Moos, R. H., \& Schaefer, J. A. (1996). Coping, stress resistance, and growth: Conceptualizing adaptive functioning. In M. Zeidner \& N. Endler (Eds.), Handbook of coping: Theory, research and application (pp. 23-43). New York: John Wiley \& Sons.

Jarro, R., Salum, G., Silva, C., Costa, M., Salles, J, \& Mandro, G. (2012). Anxiety disorders in adolescence are associated with impaired facial expression recognition to negative valence. Journal of Psychiatric Research, 46, 147-151.

Kochanska, G., Murray, K. T., \& Harlan, E. T. (2000). Effortful control in early childhood: Continuity and change, antecedents, and implications for social development. Developmental Psychology, $36,220-232$. 
Letcher, P., Sanson, A., Smart, D., \& Toumbourou J. W. (2012). Precursors and correlates of anxiety trajectories from late childhood to late adolescence. Clinical Child and Adolescent Psychology, $41,417-432$.

Lima, L., Lemos, M. S., \& Guerra, M. P. (2002). Estudo das qualidades psicométricas do SCSI (Scoolgers' Coping Strategies Inventory) numa população portuguesa. Análise Psicológica, 4 (XX), 555-570.

Lonigan, C. J., \& Philips, B. M. (2001). Temperamental influences on the development of anxiety disorders. In M. W. Vasey \& M. R. Dadds (Eds.), The developmental psychopathology of anxiety (pp. 60-91). New York: Oxford University Press.

Marzocchi, G. M., Capron, C., Pietro, M. P., Tauleria, E. D., Duyme, M., Frigerio, A.,.... Thérond C., (2004). The use of the Strengths and Difficulties Questionnaire (SDQ) in Southern European countries. European Child \& Adolescent Psychiatry [Suppl 2], 13, II/40-II/46.

McClure, E. B. (2000). A meta-analytic review of sex differences in facial expression processing and their development in infants, children, and adolescents. Psychological Bulletin, 126(3), 424:453.

Merikangas, K. R., He, J.P., Burstein, M., Swanson, S. A., Avenevoli, S., Cui, L., ... Swendsen J., (2010). Lifetime Prevalence of Mental Disorders in U.S. Adolescents: Results from the National Comorbidity Survey Replication- Adolescent Supplement (NCS-A). Journal of the American Academy of Child \& Adolescent Psychiatry, 49(10), 980-989.

Muñoz, L. C. (2009). Callous-unemotional traits are related to combined deficits in recognizing afraid faces and body poses. Journal of the American Academy of Child and Adolescent Psychiatry, $48,554-562$

Muris, P., \& Meesters, C. (2009). Reactive and Regulative Temperament in Youths: Psychometric Evaluation of the Early Adolescent Temperament Questionnaire-Revised. Journal of Psychopathology and Behavioral Assessment, 31, 7-19.

Muris, P., \& Ollendick, T. H. (2005). The role of temperament in the etiology of child psychopathology. Clinical Child and Family Psychology Review, 8, 271-289.

Nigg, J. T. (2006). Temperament and developmental psychopathology. Journal of Child Psychology and Psychiatry, 47, 395-422.

Pitzer, M., Jennen-Steinmetz, C., Esser, G., Schmidt, M. H., \& Laucht, M. (2011). Prediction of preadolescent depressive symptoms from child temperament, maternal distress, and gender: results of a prospective, longitudinal study. Development and Behavior Pediatrics, 32, 18-26.

Raimundo, R. C., \& Pinto, M. A. (2006). Stress e estratégias de coping em crianças e adolescentes em contexto escolar. Altheia, 24, 9-19.

Rothbart, M. K., \& Rueda, M. R (2005). The development of effortful control. In U. Mayr, E. Awh, \& S. W. Keele (Eds.), Developing individuality in the human brain: A tribute to Michael I. Posner (pp. 167-188). Washington, DC: American Psychological Association.

Rothbart, M., \& Bates, J. (2006). Temperament. In N. Eisenberg, W. Damon, \& R. M. Lerner (Eds.), Handbook of child psychology: Vol. 3. Social, emotional and personality development $\left(6^{\text {th }}\right.$ ed., pp. 99-166). New York: John Willey \& Sons.

Roy, B. V., Groholt, B., Heyerdahl, S., \& Clench-Aas, J. (2006). Self-report strengths and difficulties in a large Norwegian population 10-19 years: Age and gender specific results of the extended SDQ-questionnaire. European Child \& Adolescent Psychiatry, 15, 189-198.

Ryan-Wenger, N. (1990). Development and psychometric properties of the schoolager's coping strategies inventory. Nursing Research, 39(6), 344-349.

Schepman, K., Taylor, E., Collishaw, S., \& Fombonne, E. (2012). Face emotion processing in depressed children and adolescents with and without comorbid conduct disorder. Journal of Abnormal Child Psychology, 40(4), 583-593. 
Tottenham, N., Hare, T. A., \& Casey, B. J. (2011). Behavioral assessment of emotion discrimination, emotion regulation, and cognitive control in childhood, adolescence, and adulthood. Frontiers in Psychology, 2(39), 1-9.

Young, E. L., Sabbah, H., Y., Young, B. J., Reiser, M. L., \& Richardson, M. J. (2010). Gender differences and Similarities in a Screening Process for Emotional and Behavioral Risks in Secondary Schools. Journal of Emotional and Behavioral Disorders, 18(4), 225-235.

Zalewski, M., Lengua, L. J., Wilson, A. C., Trancik, A., \& Bazinet, A. (2011a). Associations of coping and appraisal styles with emotion regulation during preadolescence. Journal of Experimental Child Psychology, 110, 141-158.

Zalewski, M., Lengua, L.. J., Wilson, A. C, Trancik, A., \& Bazinet, A. (2011b). Emotion regulation profiles, temperament, and adjustment problems in preadolescents. Child Development, 82, 951-966. 